\title{
Cellular senescence and the senescent secretory phenotype: therapeutic opportunities
}

\author{
Tamara Tchkonia, ${ }^{1}$ Yi Zhu, ${ }^{1}$ Jan van Deursen, ${ }^{1}$ Judith Campisi, ${ }^{2}$ and James L. Kirkland ${ }^{1}$
}

${ }^{1}$ Robert and Arlene Kogod Center on Aging, Mayo Clinic, Rochester, Minnesota, USA. Buck Institute for Research on Aging, Novato, California, USA.

\begin{abstract}
Aging is the largest risk factor for most chronic diseases, which account for the majority of morbidity and health care expenditures in developed nations. New findings suggest that aging is a modifiable risk factor, and it may be feasible to delay age-related diseases as a group by modulating fundamental aging mechanisms. One such mechanism is cellular senescence, which can cause chronic inflammation through the senescence-associated secretory phenotype (SASP). We review the mechanisms that induce senescence and the SASP, their associations with chronic disease and frailty, therapeutic opportunities based on targeting senescent cells and the SASP, and potential paths to developing clinical interventions.
\end{abstract}

\section{Introduction}

The world's elderly population is growing rapidly. As people reach advanced age, they often face years of disability marked by multiple chronic diseases, frailty, and loss of independence. This burden of disability threatens social and economic stability. A huge challenge for modern biomedical research is to compress, if not eliminate, this period of frailty and disability and to increase health span. How can this challenge be met?

Aging is a large, if not the leading, risk factor for most of the chronic conditions that limit survival, independence, and well-being (1). These chronic disorders, including atherosclerosis, most cancers, dementias, diabetes, and many others (Figure 1), become progressively more prevalent as the elderly population grows. A prime suspected cause of these prominent age-related disorders is the chronic, nonmicrobial inflammation that develops in multiple tissues. Hallmarks of inflammation, including elevated IL-6, TNF$\alpha$, and immune cell chemokines, are associated with dementias (2), depression (3), atherosclerosis (4-8), cancers (9-11), diabetes (12$14)$, and mortality $(2,15,16)$. Inflammation is perhaps the most important physiologic correlate of the age-related frailty syndrome (17-20), which includes heightened vulnerability to stresses (e.g., surgery, infection, or trauma), coupled with muscle wasting (sarcopenia) and cachexia/fat tissue loss, all of which become increasingly common in old age (17-19, 21-29). Frailty predisposes to chronic disease, loss of independence, and mortality and greatly increases health costs $(25,27)$.

Until recently, the powerful association between age and chronic disease has mainly been noted with little hope of intervention. A critical roadblock to enhancing health span is the lack of effective treatments for age-related frailty and chronic diseases as a group. Currently available treatments (social supports, mobility aides, and "Band-Aid" treatments for end-stage, downstream symptoms) are not directed at the root causes of age-related dysfunction. Treating chronic diseases one at a time does not suffice (30). Calculations based on mortality data in the United States produce surprising predictions: if cancer was eliminated as a cause of death, average human life span would increase only $3 \%-4 \%$ (31). The same is true were ischemic heart disease to be "cured" (30). Yet caloric restriction, which retards broad basic aging processes by as yet

Conflict of interest: The authors have declared that no conflict of interest exists. Citation for this article: J Clin Invest. 2013;123(3):966-972. doi:10.1172/JCI64098. incompletely understood mechanisms, extends life span in animal models, including mice, by much larger increments (32). Clearly, clinical practice would be transformed if mechanism-based treatments could be devised that break the link between fundamental aging processes and chronic diseases, making aging a modifiable risk factor. The recent awareness that age-related disorders can be driven by one or more basic aging processes has inspired efforts to identify these processes and develop strategies, preferably pharmacological in nature, to intervene.

\section{Cellular senescence}

One basic process that may contribute to age-related dysfunction and chronic sterile inflammation is cellular senescence (Figure 2). Cellular senescence refers to the essentially irreversible growth arrest that occurs when cells experience potentially oncogenic insults (33-38). There is now strong evidence that cellular senescence is a potent anticancer mechanism (39-42). In contrast, despite its name, its discovery over 50 years ago, and increasing data associating senescent cells with aging phenotypes and age-related pathology (43-50), evidence has only recently emerged showing that eliminating senescent cells can actually delay age-related dysfunction (51), at least in a progeroid mouse model. This finding still must be tested in chronologically aged models, but this is the first clear evidence that senescent cells are important drivers of multiple age-related pathologies. How cellular senescence promotes age-related diseases, frailty, and dysfunction remains one of the important questions in the biology of aging and clinical geriatrics.

The abundance of senescent cells increases in multiple tissues with chronological aging and in progeroid syndromes (43, 47, 50, 52-55). Several processes have been identified that cause or are associated with cellular senescence, all of which also increase with age (Figure 3). Senescence-causing inducers include repeated cell division and strong mitogenic signals, telomere shortening, DNA damage and mutations, protein aggregation, and increased ROS $(46,56-60)$. These insults activate the $\mathrm{p} 53$ and $\mathrm{p} 16^{\mathrm{INK} 4 \mathrm{a}}$ tumor suppressor pathways and potentially other pathways that initiate a senescence response. Once initiated, senescence takes days to weeks to become fully established and irreversible. The process is reinforced by an intracellular signaling loop including ROS linked to DNA damage responses (DDRs), NFKB, and transforming growth factor- $\beta$, as well as an IL- $1 \alpha$, IL- 6 , and CCAAT 


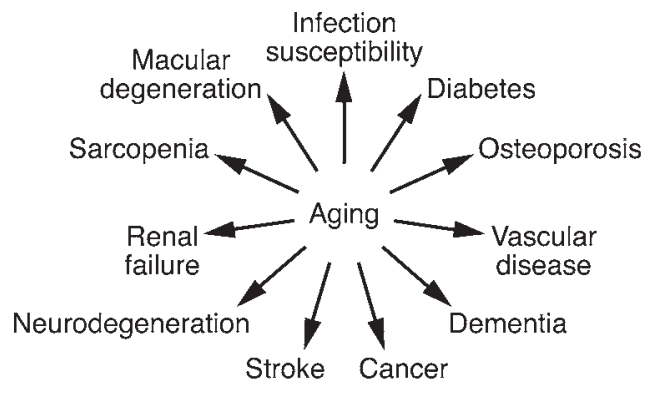

\section{Figure 1}

Aging is the leading risk factor for most serious chronic diseases and disabilities, including strokes, heart disease, cancers, dementias, osteoporosis, arthritis, diabetes, metabolic syndrome, kidney failure, blindness, and frailty.

enhancer binding protein- $\beta$ (C/EBP- $\beta$ ) loop (59, 61-64). Senescent cells reorganize chromatin, resulting in heterochromatin formation, extensive gene expression changes, increased cell size and protein content, and changes in cell and organelle shape $(55,65$, 66). Senescent cells are metabolically active, relatively resistant to apoptosis, and appear to be removed by the immune system (33, $67,68)$. Senescence is effectively a cell fate that, like replication, differentiation, or apoptosis, can occur at any point in life.

A common feature of aging tissues is low-level chronic inflammation, termed sterile inflammation (indicating an absence of detectable pathogens) or inflammaging (69-72). Chronic inflammation can drive pathology by at least two mechanisms. First, infiltrating immune cells can degrade tissues because they release reactive or toxic moieties. Second, inflammatory cytokines can provoke phenotypic changes that are independent of the immune system in nearby cells. For example, IL- 6 and IL-8 can stimulate angiogenesis, disrupt cell-cell communication, impede macrophage function, induce innate immune responses, and promote epithelial and endothelial cell migration and invasion (73-79).

Little is known about the source of the sterile inflammation that fuels most major age-related diseases, including degenerative pathologies, such as loss of brain function, as well as hyperproliferative pathologies, such as cancer. This chronic inflammation may derive partly from an age-related decline in homeostatic immune function or resistance to endogenous microbes. Chronic inflammation may also derive in part from senescent cells: senescent cells secrete proinflammatory cytokines, chemokines, and proteases, termed the senescence-associated secretory phenotype (SASP) $(80,81)$.

\section{SASP}

The SASP is primarily a DDR (82). The SASP, through the inflammatory, growth-promoting, and remodeling factors that it produces, can potentially explain how senescent cells alter tissue microenvironments, attract immune cells, and, ironically, induce malignant phenotypes in nearby cells. Proteins that are associated with the SASP, such as TNF- $\alpha$, IL-6, MMPs, monocyte chemoattractant protein-1 (MCP-1), and IGF binding proteins (IGFBPs), increase in multiple tissues with chronological aging (64), and occur in conjunction with sterile inflammation. Furthermore, expression of the SASP components, IL-6, IGFBP-2, and plasminogen activator inhibitor-1, is much higher in p16 ${ }^{\text {INK4a }}$-positive senescent cells isolated directly from fat tissue of older progeroid mice compared with nonsenescent cells from the same tissue (51). This finding suggests that the SASP is the main driver of age-related inflammation, at least in fat tissue under certain conditions. Thus, selective elimination of senescent cells or their effects might be a means to reduce age-related sterile chronic inflammation, enhance health span, and interrupt the link between aging and chronic disease.

Cellular senescence is a double-edged sword. Central to understanding how senescent cells could have positive and negative effects on organismal health are two concepts. The first is antagonistic pleiotropy. This element of the evolutionary theory of aging predicts the existence of processes that were selected to ensure early life fitness (e.g., protection from cancer by promoting immune responses), but have unselected deleterious effects late in life that cause aging phenotypes and pathology (83).

The second concept is the SASP, which can have positive or negative effects, depending on context. The SASP can cause local and potentially systemic inflammation, disrupt tissue architecture, and stimulate growth of nearby malignant cells when the SASP is both pronounced or persistent, as in old age, massive obesity, or progerias $(65,84-88)$. Conversely, a localized, time-limited SASP may be important in resolving tissue damage, at least in younger individuals, an example of antagonistic pleiotropy. The SASP might alert nearby cells to potential danger and promote immune clearance of the damaged cells $(67,68,89)$. Furthermore, SASP MMPs limit fibrosis following liver injury or during skin wound healing $(68,90,91)$, which is beneficial. The SASP cytokines, IL-6 and IL-8, also reinforce the senescence growth arrest, at least in some senescent cells $(62,63)$, which is beneficial in defense against cancer. However, these cytokines can also cause epithelial-to-mesenchymal

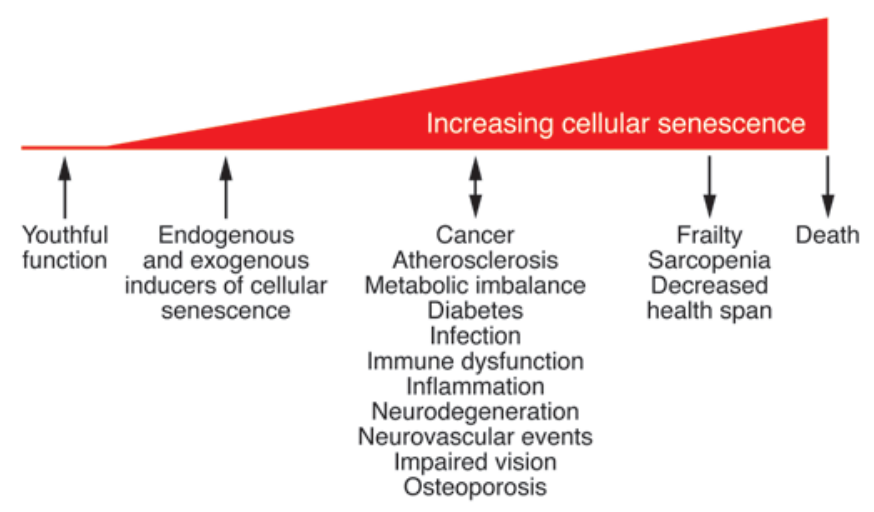

\section{Figure 2}

A disruption of the intersection between fundamental aging mechanisms and processes that lead to chronic diseases may delay agerelated diseases and disabilities as a group and thereby lengthen health span. The increasing burden of senescent cells might contribute to the early etiology of age-related diseases and accelerate progression of these diseases following their initiation. Chronic disease pathology, coupled with the spread of senescence to neighboring healthy cells, might further drive cellular senescence, thus contributing to a spiral of increasing inflammation and dysfunction. Among other possibilities, chronic inflammation associated with the SASP, combined with with inflammation from preclinical and overt chronic disease, may predispose to frailty, sarcopenia, and eventually mortality. 


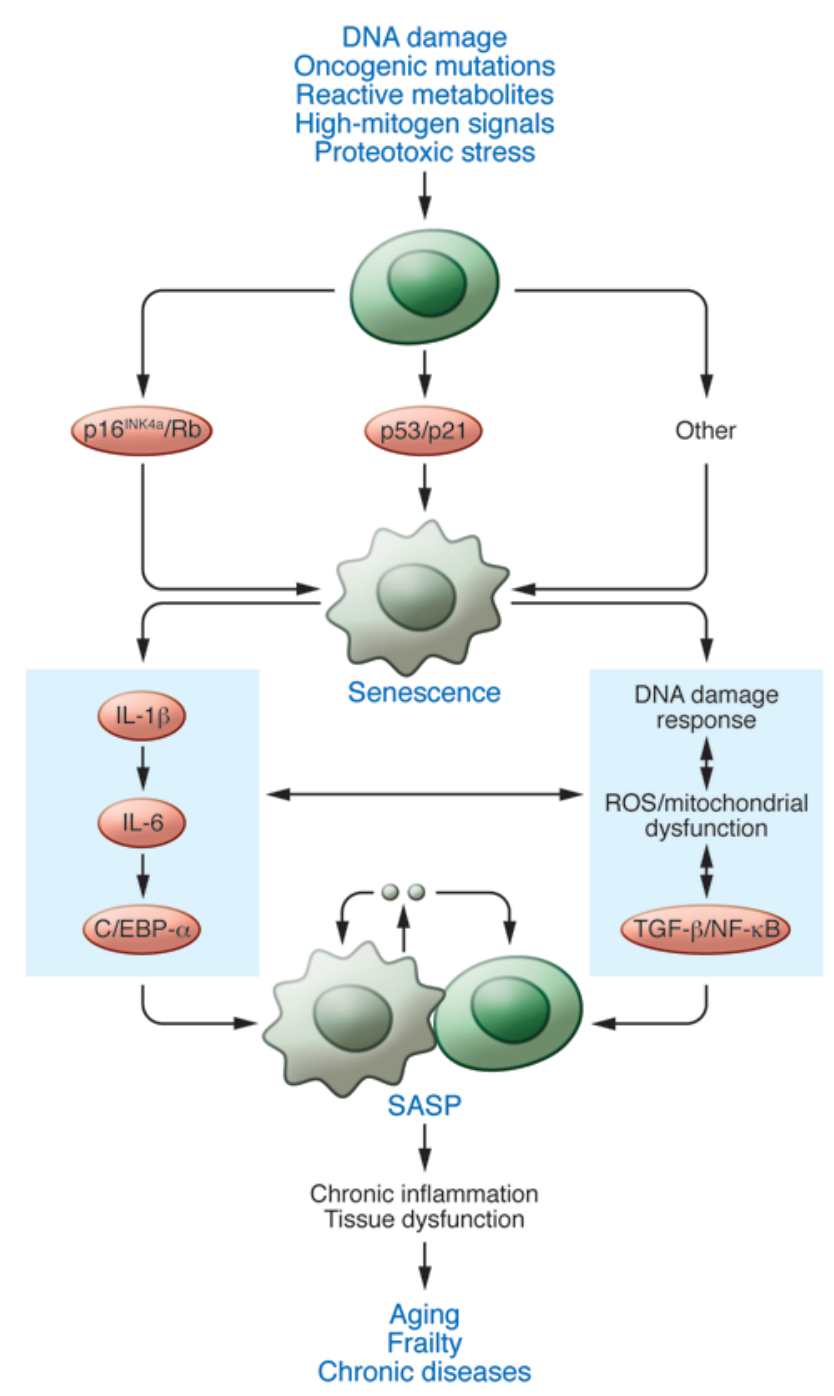

transitions, which promote cancer $(77,78)$. Conceivably, the SASP could play a role in defense against infections or other insults, possibilities that need to be studied. Remarkably, though, continuous clearance of senescent cells for over 9 months did not have overt adverse effects, but rather enhanced health span and function, at least in progeroid mice housed in an animal facility (51).

An intracellular IL-1 $\alpha$ / miR-146a/b / IL-6 / C/EBP- $\beta$ loop as well as related $\mathrm{p} 38 / \mathrm{NF}-\kappa \mathrm{B}-$ and $\mathrm{mTOR}-$ mediated pathways appear to contribute to the changes in gene expression that result in the SASP (Figure 2 and refs. 61-64, 92-94). SASP components include proteins that are generally conserved across cell types, although differences exist among cell types (81) and presumably across tissues, a speculation that needs further testing. Very little is known about the contribution, if any, of nonprotein factors such as nucleotides, bradykines, prostenoids, ceramides, or ROS to effects of the SASP. The composition of the SASP may vary as time progresses after the initiation of senescence and might partly depend on the mechanism through which senescence is induced. In support of this possibility, senescence induced by oncogenic RAS, p $16^{\text {INK } 4 a}$ overexpression, or $\mathrm{p} 53$ activity is associated with variability in both the quality and extent of SASP protein secretion $(81,95)$. Thus, while we speak of the SASP, it is unlikely that there is a singular

\section{Figure 3}

A number of inducers can act alone or in combination to push cells into the senescent cell fate through pathways involving p16 INK4a/Rb, p53/p21, and likely other pathways. Triggers may include DNA damage (e.g., telomere shortening and single- and double-strand breaks); oncogenic mutations (e.g., Ras, Myc, B-Raf); reactive metabolites (e.g., ROS, ceramides, fatty acids, high glucose); high mitogen and nutrient signals that increase mTOR activity; and proteotoxic stress (e.g., protein aggregation and unfolded proteins). These may contribute to widespread changes in gene expression and chromatin remodeling (heterochromatin formation) that underlie senescence-associated growth arrest, the SASP, and changes in morphology. In these respects, cellular senescence can be viewed as a cell fate reminiscent of differentiation, replication, or apoptosis (external and internal inducers, transcription factor cascades, gene expression changes and chromatin remodeling, leading to changes in function). Evidence is better for some of the initiators and mediators of senescence than for others, and future research is likely to uncover additional initiators and mediators. Intracellular autocrine loops reinforce progression to irreversible replicative arrest, heterochromatin formation, and initiation of the SASP over a matter of days to weeks. In addition to removing cells from the progenitor/stem cell pool, senescence may contribute to tissue dysfunction and chronic disease predisposition through the SASP and associated chronic sterile inflammation and degradation of the extracellular matrix. 
including MCP-1, are prominent components of the SASP (64, 81). However, tissue macrophage responses appear to decline with aging (101), potentially contributing to senescent cell accumulation in old age. A high burden of senescent cells could interfere with immune function. Consistent with this speculation, chronic exposure to IL-6 inhibits macrophage function (102) and SASP proteases could cleave FAS ligand or other cell surface proteins required for effective immune function. More insight is needed into how senescent cells affect the immune system and are removed by it. The speculation that reducing senescent cell burden could actually enhance immune responses to pathogens needs to be tested.

Interventions targeting cellular senescence and the SASP Potential strategies for mitigating the deleterious effects of senescent cells include interfering with pathways that lead to senescence-associated growth arrest, eliminating senescent cells, and interfering with the adverse effects of senescent cells by targeting the SASP. The first strategy could be problematic if the mechanisms through which cellular senescence defends against cancer are compromised. Interfering with pathways that can induce senescence-associated replicative arrest are likely to promote cancer, as occurs when $\mathrm{p} 16^{\text {INK4a }}$, the retinoblastoma $(\mathrm{Rb})$ protein, or p53 is diminished or inactivated $(67,103)$. On the other hand, strategies that delay senescent cell accumulation by reducing progenitor turnover, metabolic damage, or other processes that can cause cell damage might be beneficial. For example, caloric restriction delays cellular senescence (104) and could exert part of its beneficial effect on health span through doing so, a speculation that merits further testing.

The second approach, eliminating already-formed senescent cells, may not only reduce tissue inflammation and organ dysfunction but might also reduce cancer risk. Cells can become senescent when they harbor potentially oncogenic mutations. Furthermore, senescent cells can promote malignant transformation in neighboring cells in mouse xenografts (86), possibly through SASP-induced inflammation and tissue MMP and mitogen secretion. Indeed, health span was enhanced by clearing senescent cells in progeroid mice, without interfering with anticarcinogenic pathways upstream of senescence (51). Because terminally differentiated cells share some properties of senescent cells such as growth arrest, we were concerned that targeting senescent cells in INK-ATTAC mice might damage cells like neurons. However, terminally differentiated cells did not appear to be disrupted to the point of causing symptoms, even after over a year of continuous senescent cell removal.

The third approach, preventing development of the SASP or ameliorating its effects, could also enhance function and reduce inflammation and cancer risk, albeit without the finality of actually eliminating the senescent cells that potentially harbor cancerous lesions. Ideally, ameliorating the SASP would be achieved without interfering with senescence-associated anti-oncogenic pathways or undoing growth arrest.

Eliminating senescent cells may be possible in humans. The distinct morphology, secreted protein patterns, and gene expression profiles of senescent cells support the feasibility of doing so. At least two approaches can be envisaged for removing senescent cells: the use of antibodies to specifically target senescent cells or the development of small molecules to selectively kill them. Other approaches might include viral-based nucleotide delivery or vaccines. The antibody approach would entail developing biologicals that recognize epitopes that are more highly expressed by senescent compared with nonsenescent cells, coupled to a cytolytic agent. This approach has been difficult in the cancer field and could also be problematic with respect to eliminating senescent cells. However, unlike for cancer interventions, complete elimination of senescent cells may not be necessary for achieving beneficial effects. Thus, targeting epitopes that differ quantitatively between senescent and nonsenescent cells may be reasonable, without needing to discover epitopes that are uniquely present on senescent cells.

The small-molecule approach could be based on high-throughput, cell-based screens of chemical libraries to discover "druggable" molecules that kill senescent cells with greater selectivity than nonsenescent cells. Alternatively, molecular target-based high-throughput screens focused on pathways identified through expression, proteomic, or metabolomic analyses of senescent versus nonsenescent cells may work. These small-molecule approaches could also be adapted to targeting the SASP, as opposed to killing senescent cells. Using one of these approaches, it was discovered that glucocorticoids ameliorate the SASP (105).

Developing interventions to target senescent cells or the SASP will be a tall order. Possibly, combinations of approaches will be required. Because senescent cells do not divide, they are unlikely to develop drug resistance, a problem encountered with compounds that target dividing cancer cells or microbes. Also, finding compounds or antibodies that target $100 \%$ of senescent cells and $0 \%$ of normal cells might not be necessary to achieve clinical benefit. Thus, it may be feasible to discover agents that target senescent cells or the SASP and test them in aged animals to determine whether they reproduce the beneficial effects of removing senescent cells from genetically engineered progeroid mice.

\section{Clinical trials strategies}

Designing clinical trials to test the effectiveness of agents that target senescent cells is a challenge (106). Clearly, studying effects on human life span or health span is not feasible within a realistic time frame. Drugs that must be given early in life to have a beneficial effect in old age would also be nearly impossible to translate into clinical application. Such drugs would need to have virtually no side effects over years to decades. The time required for clinical trials would be prohibitive. Senolytic drugs would need to be developed for subjects who already have symptoms or who are guaranteed to develop them within a short time (e.g., patients with progerias or members of families who carry mutations causing early-onset Alzheimer's disease that have essentially complete penetrance). Readily measurable outcome parameters would need to be devised and accepted by regulatory authorities.

We envisage several potential clinical trial scenarios. Based on these, it may be reasonable to work backward to design drug screens and animal testing strategies. One scenario would be to target specific age-related chronic diseases in which senescence plays an etiologic role and in which eliminating senescent cells or SASP components would result in a rapidly detectable, clinically meaningful response. For example, effects of senolytics on ameliorating peripheral insulin resistance in obese subjects could be tested because massive obesity is associated with fat tissue senescent cell accumulation and elevated levels of circulating SASP-related cytokines that cause insulin resistance $(56,85)$. Another example would be to test whether progression of cancers that have failed conventional treatment is prevented, based on the contribution of senescent cells to cancer progression in xenograft 
models (86). Other possibilities include testing whether senolytics enhance the resolution of atherosclerotic lesions, osteoarthritis, or fracture nonunion, conditions that are associated with focal senescent cell accumulation (107-109). These conditions might be amenable to local treatment, possibly by injection of senolytic agents. The effects of senolytics on the progression of dementia could be tested in longer clinical trials (lasting months rather than weeks) because Alzheimer's disease and other dementias are associated with senescent cell accumulation at sites of brain pathology (reviewed in ref. 110). Of course, all these approaches would first need to be thoroughly vetted in proof-of-principle, preclinical studies in appropriate disease-specific animal models (preferably in old rather than young genetically modified animals, so that the aging tissue microenvironment is reproduced).

Another scenario is to target the frailty syndrome, which is associated with systemic inflammation (17-20) and potentially the SASP. Trials can be envisaged in borderline frail subjects (with intermediate Fried or Rockwood scores; refs. 25, 27, 28) who are about to undergo medical procedures that increase inflammation and/or cellular senescence and that might push subjects into overt frailty or delirium. These procedures might include, for example, chemotherapy (particularly DNA-damaging agents), radiotherapy, bone marrow transplantation, and anesthesia/elective surgery. In these situations, senolytic treatment for a period before (but not during) the medical procedure might have a measurable effect on time to recovery of symptom scores, strength, or cognition. These possibilities need to be tested first in animal models such as the INK-ATTAC mouse, in which senescent cells can be quantified because of a fluorescent marker and can be selectively eliminated in an inducible manner (51).

\section{Research issues}

Issues that need to be addressed before moving senolytics into clinical application include the development of biomarkers of senescent cell burden/SASP activity, identification of any adverse consequences of targeting senescent cells, and determination of when to begin treatment and whether intermittent or continuous treatment is optimal. Blood biomarkers of senescent cell burden, perhaps based on detecting SASP products, would facilitate clinical trials. These biomarkers could be calibrated using a gold standard - for example, INK-ATTAC mice that have had senescent cells removed, senescent cell abundance in fat tissue biopsies, or whole body senescent cell counts at necropsy. Thor- ough testing of whether senescent cell removal impairs response to infection, wound healing, scarring, or other functions must be conducted in animals and eventually humans. Intermittent rather than continuous senescent cell elimination during periods of relatively good health could remove potentially precancerous senescent cells and reduce chronic inflammation due to the SASP, yet minimize potential adverse effects of senescent cell clearance. This approach might be more attractive than ameliorating the SASP, which may need to be done continuously, unlike removal of senescent cells.

Presumably, clinical trials of senolytics will be done in elderly subjects as well as younger subjects with diseases associated with cellular senescence and inflammation, such as diabetes in massive obesity. However, elderly subjects are not often included in clinical trials, and their study presents special clinical and logistical challenges. There are few geriatricians who have both a deep familiarity with the basic biology of aging and experience in conducting trials of investigational new drugs (INDs). Further, few clinical trial investigators with IND experience have training in geriatrics and a deep understanding about the particular needs of elderly subjects or outcomes relevant to elderly populations. There is an urgent need to build teams of basic scientists in the biology of aging, geriatricians, and clinical trial investigators. In the longer term, there is a need to train investigators who can lead efforts to translate findings from the basic biology of aging into clinical application (106).

\section{Conclusion}

If the premise is correct that targeting senescent cells or the SASP can delay or prevent multiple age-related chronic diseases as a group, rather than one at a time, and if this premise can be translated into clinical treatments, we are cautiously optimistic that health care as we know it might be transformed.

\section{Acknowledgments}

The authors are grateful for administrative and editing assistance from L. Wadum and J. Armstrong. This work was supported by NIH grant AG041122, and by the Noaber Foundation and the Ellison Medical Foundation.

Address correspondence to: James L. Kirkland, Robert and Arlene Kogod Center on Aging, Mayo Clinic, Guggenheim 7-01, 200 First Street, S.W., Rochester, Minnesota 55905, USA. Phone: 507.266.9151; Fax: 507.293.3853; E-mail: kirkland.james@mayo.edu.

\footnotetext{
1. [No authors listed]. The Silver Book: Chronic Disease and Medical Innovation in an Aging Nation. Washington, DC, USA: Alliance for Aging Research; 2012.

2. Bruunsgaard H, Pedersen BK. Age-related inflammatory cytokines and disease. Immunol Allergy Clin North Am. 2003;23(1):15-39.

3. Howren MB, Lamkin DM, Suls J. Associations of depression with C-reactive protein, IL-1, and IL-6: a meta-analysis. Psychosom Med. 2009;71(2):171-186.

4. Tuomisto K, Jousilahti P, Sundvall J, Pajunen P, Salomaa V. C-reactive protein, interleukin- 6 and tumor necrosis factor alpha as predictors of incident coronary and cardiovascular events and total mortality. A population-based, prospective study. Thromb Haemost. 2006;95(3):511-518.

5. Margolis KL, et al. Leukocyte count as a predictor of cardiovascular events and mortality in postmenopausal women: the Women's Health Initiative Observational Study. Arch Intern Med. 2005;165(5):500-508

6. Brown DW, Giles WH, Croft JB. White blood cell
}

count: an independent predictor of coronary heart disease mortality among a national cohort. J Clin Epidemiol. 2001;54(3):316-322.

7. Cesari $\mathrm{M}$, et al. Inflammatory markers and cardiovascular disease (The Health, Aging and Body Composition [Health ABC] Study). Am J Cardiol. 2003;92(5):522-528.

8. Pai JK, et al. Inflammatory markers and the risk of coronary heart disease in men and women. $N$ EnglJ Med. 2004;351(25):2599-2610.

9. O'Connor PM, Lapointe TK, Beck PL, Buret AG. Mechanisms by which inflammation may increase intestinal cancer risk in inflammatory bowel disease. Inflamm Bowel Dis. 2010;16(8):1411-1420.

10. Schetter AJ, Heegaard NH, Harris CC. Inflammation and cancer: interweaving microRNA, free radical, cytokine and p53 pathways. Carcinogenesis. 2010;31(1):37-49.

11. Srikrishna G, Freeze HH. Endogenous damage-associated molecular pattern molecules at the crossroads of inflammation and cancer. Neoplasia.
2009;11(7):615-628.

12. Spranger J, et al. Inflammatory cytokines and the risk to develop type 2 diabetes: results of the prospective population-based European Prospective Investigation into Cancer and Nutrition (EPIC)-Potsdam Study. Diabetes. 2003;52(3):812-817.

13. Hu FB, Meigs JB, Li TY, Rifai N, Manson JE. Inflammatory markers and risk of developing type 2 diabetes in women. Diabetes. 2004;53(3):693-700.

14. Pradhan AD, Manson JE, Rifai N, Buring JE, Ridker PM. C-reactive protein, interleukin 6, and risk of developing type 2 diabetes mellitus. J Amer Med Assoc. 2001;286(3):327-334.

15. Harris TB, et al. Associations of elevated interleukin-6 and C-reactive protein levels with mortality in the elderly. Am J Med. 1999;106(5):506-512.

16. Bruunsgaard H, Andersen-Ranberg K, Hjelmborg JB, Pedersen BK, Jeune B. Elevated levels of tumor necrosis factor alpha and mortality in centenarians. Am J Med. 2003;115(4):278-283.

17. Kanapuru B, Ershler WB. Inflammation, coag- 
ulation, and the pathway to frailty. Am J Med. 2009;122(7):605-613

18. Walston J, et al. Frailty and activation of the inflammation and coagulation systems with and without clinical comorbidities: results from the Cardiovascular Health Study. Arch Intern Med. 2002;162(20):2333-2341.

19. Leng SX, Xue QL, Tian J, Walston JD, Fried LP. Inflammation and frailty in older women. $J \mathrm{Am}$ Geriatr Soc. 2007;55(6):864-871.

20. Ferrucci L, et al. Serum IL-6 level and the development of disability in older persons. J Am Geriatr Soc. 1999;47(6):639-646.

21. Bandeen-Roche K, Walston JD, Huang Y, Semba RD, Ferrucci L. Measuring systemic inflammatory regulation in older adults: evidence and utility. Rejuvenation Res. 2009;12(6):403-410.

22. Qu T, et al. Upregulated ex vivo expression of stress-responsive inflammatory pathway genes by LPS-challenged CD14(+) monocytes in frail older adults. Mech Ageing Dev. 2009;130(3):161-166.

23. Walston JD, et al. Inflammation and stress-related candidate genes, plasma interleukin- 6 levels, and longevity in older adults. Exp Gerontol. 2009;44(5):350-355.

24. Walston J, et al. Research agenda for frailty in older adults: toward a better understanding of physiology and etiology: summary from the American Geriatrics Society/National Institute on Aging research conference on frailty in older adults. $J \mathrm{Am}$ Geriatr Soc. 2006;54(6):991-1001.

25. Fried LP, et al. Frailty in older adults: evidence for a phenotype. J Gerontol A Biol Sci Med Sci. 2001;56(3):M146-M156.

26. Bandeen-Roche K, et al. Phenotype of frailty: characterization in the women's health and aging studies. J Gerontol A Biol Sci Med Sci. 2006;61(3):262-266.

27. Rockwood K, Mitnitski A, Song X, Steen B, Skoog I. Long-term risks of death and institutionalization of elderly people in relation to deficit accumulation at age 70. J Am Geriatr Soc. 2006;54(6):975-979.

28. Rockwood K, Mitnitski A. Frailty defined by deficit accumulation and geriatric medicine defined by frailty. Clin Geriatr Med. 2011;27(1):17-26.

29. Lucicesare A, Hubbard RE, Searle SD, Rockwood K. An index of self-rated health deficits in relation to frailty and adverse outcomes in older adults. Aging Clin Exp Res. 2010;22(3):255-260.

30. Fried LP, et al. Nonlinear multisystem physiological dysregulation associated with frailty in older women: implications for etiology and treatment. J Gerontol A Biol Sci Med Sci. 2009;64(10):1049-1057.

31. Olshansky SJ, Carnes BA, Cassel C. In search of Methuselah: estimating the upper limits to human longevity. Science. 1990;250(4981):634-640.

32. Kapahi P, et al. With TOR, less is more: a key role for the conserved nutrient-sensing TOR pathway in aging. Cell Metab. 2010;11(6):453-465.

33. Campisi J, d'Adda di Fagagna F. Cellular senescence: when bad things happen to good cells. Nat Rev Mol Cell Biol. 2007;8(9):729-740.

34. Suram A, et al. Oncogene-induced telomere dysfunction enforces cellular senescence in human cancer precursor lesions. EMBOJ. 2012;31(13):2839-2851.

35. Chen JH, Stoeber K, Kingsbury S, Ozanne SE, Williams GH, Hales CN. Loss of proliferative capacity and induction of senescence in oxidatively stressed human fibroblasts. J Biol Chem. 2004;279(47):49439-49446.

36. Courtois-Cox S, Jones SL, Cichowski K. Many roads lead to oncogene-induced senescence. Oncogene. 2008;27(20):2801-2809.

37. Braig M, et al. Oncogene-induced senescence as an initial barrier in lymphoma development. Nature. 2005;436(7051):660-665.

38. Haigis KM, Sweet-Cordero A. New insights into oncogenic stress. Nat Genet. 2011;43(3):177-178.

39. Braig M, Schmitt CA. Oncogene-induced senes- cence: putting the brakes on tumor development. Cancer Res. 2006;66(6):2881-2884.

40. Prieur A, Peeper DS. Cellular senescence in vivo: a barrier to tumorigenesis. Curr Opin Cell Biol. 2008;20(2):150-155.

41. Campisi J. Cellular senescence as a tumor-suppressor mechanism. Trends Cell Biol. 2001;11(11):S27-S31.

42. Guerra C, et al. Pancreatitis-induced inflammation contributes to pancreatic cancer by inhibiting oncogene-induced senescence. Cancer Cell. 2011;19(6):728-739.

43. Burton DG. Cellular senescence, ageing and disease. Age (Dordr). 2009;31(1):1-9.

44. Campisi J. Senescent cells, tumor suppression, and organismal aging: good citizens, bad neighbors. Cell. 2005;120(4):513-522

45. Collado M, Blasco MA, Serrano M. Cellular senescence in cancer and aging. Cell. 2007;130(2):223-233.

46. Jeyapalan JC, Sedivy JM. Cellular senescence and organismal aging. Mech Ageing Dev. 2008; 129(7-8):467-474.

47. Baker DJ, et al. Opposing roles for p16Ink4a and p19Arf in senescence and ageing caused by BubR1 insufficiency. Nat Cell Biol. 2008;10(7):825-836.

48. Krishnamurthy J, et al. Ink4a/Arf expression is a biomarker of aging. J Clin Invest. 2004; 114(9):1299-1307.

49. Jeyapalan JC, Ferreira M, Sedivy JM, Herbig U. Accumulation of senescent cells in mitotic tissue of aging primates. Mech Ageing Dev. 2007;128(1):36-44.

50. Waaijer ME, et al. The number of $\mathrm{p} 16 \mathrm{INK} 4 \mathrm{a}$ positive cells in human skin reflects biological age. Aging Cell. 2012;11(4):722-725.

51. Baker DJ, et al. Clearance of p16Ink4a-positive senescent cells delays ageing-associated disorders. Nature. 2011;479(7372):232-236.

52. Herbig U, Ferreira M, Condel L, Carey D, Sedivy JM. Cellular senescence in aging primates. Science. 2006;311(5765):1257

53. Ohtani N, Yamakoshi K, Takahashi A, Hara E. Real-time in vivo imaging of p16gene expression: a new approach to study senescence stress signaling in living animals. Cell Div. 2010;5:1.

54. Hasty P, Campisi J, Hoeijmakers J, van Steeg H, Vijg $\mathrm{J}$. Aging and genome maintenance: lessons from the mouse? Science. 2003;299(5611):1355-1359.

55. Campisi J, Andersen JK, Kapahi P, Melov S. Cellular senescence: a link between cancer and age-related degenerative disease? Semin Cancer Biol. 2011;21(6):354-359.

56. Tchkonia T, et al. Fat tissue, aging, and cellular senescence. Aging Cell. 2010;9(5):667-684

57. Ksiazek K, Mikula-Pietrasik J, Olijslagers S, Jorres A, von Zglinicki T, Witowski J. Vulnerability to oxidative stress and different patterns of senescence in human peritoneal mesothelial cell strains. Am J Physiol Regul Integr Comp Physiol. 2009;296(2):R374-R382.

58. Weyemi U, et al. ROS-generating NADPH oxidase NOX4 is a critical mediator in oncogenic H-Ras-induced DNA damage and subsequent senescence. Oncogene. 2012;31(9):1117-1129.

59. Passos JF, et al. Feedback between $\mathrm{p} 21$ and reactive oxygen production is necessary for cell senescence. Mol Syst Biol. 2010;6:347.

60. Sitte N, Merker K, Von Zglinicki T, Grune T, Davies KJ. Protein oxidation and degradation during cellular senescence of human BJ fibroblasts: part I - effects of proliferative senescence. FASEB J. 2000;14(15):2495-2502

61. Kuilman T, Michaloglou C, Mooi WJ, Peeper DS. The essence of senescence. Genes Dev. 2010;24(22):2463-2479.

62. Kuilman T, et al. Oncogene-induced senescence relayed by an interleukin-dependent inflammatory network. Cell. 2008;133(6):958-961.

63. Acosta JC, et al. Chemokine signaling via the CXCR2 receptor reinforces senescence. Cell. 2008;133(6):958-961.
64. Freund A, Orjalo AV, Desprez PY, Campisi J. Inflammatory networks during cellular senescence: causes and consequences. Trends Mol Med. 2010;16(5):238-246.

65. Rodier F, Campisi J. Four faces of cellular senescence. J Cell Biol. 2011;192(4):547-556.

66. De Cecco M, Jeyapalan J, Zhao X, Tamamori-Adachi $M$, Sedivy JM. Nuclear protein accumulation in cellular senescence and organismal aging revealed with a novel single-cell resolution fluorescence microscopy assay. Aging (Albany NY). 2011;3(10):955-967.

67. Xue W, et al. Senescence and tumour clearance is triggered by $\mathrm{p} 53$ restoration in murine liver carcinomas. Nature. 2007;445(7128):656-660.

68. Krizhanovsky V, et al. Senescence of activated stellate cells limits liver fibrosis. Cell. 2008;134(4):657-667.

69. Chung HY, et al. Molecular inflammation: underpinnings of aging and age-related diseases. Ageing Res Rev. 2009;8(1):18-30.

70. Franceschi $\mathrm{C}$, et al. Inflammaging and anti-inflammaging: a systemic perspective on aging and longevity emerged from studies in humans. Mech Ageing Dev. 2007;128(1):92-105.

71. Vasto S, et al. Inflammatory networks in ageing, age-related diseases and longevity. Mech Ageing Dev . 2007;128(1):83-91.

72. Ferrucci L, Ble A, Bandinelli S, Lauretani F, Suthers K, Guralnik JM. A flame burning within. Aging Clin Exp Res. 2004;16(3):240-243.

73. Ancrile B, Lim KH, Counter CM. Oncogenic Ras-induced secretion of IL6 is required for tumorigenesis. Genes Dev. 2007;21(14):1714-1719.

74. Badache A, Hynes NE. Interleukin 6 inhibits proliferation and, in cooperation with an epidermal growth factor receptor autocrine loop, increases migration of T47D breast cancer cells. Cancer Res. 2001;61(1):383-391.

75. Desai TR, Leeper NJ, Hynes KL, Gewertz BL. Interleukin-6 causes endothelial barrier dysfunction via the protein kinase C pathway. J Surg Res. 2002;104(2):118-123.

76. Naugler WE, Karin M. The wolf in sheep's clothing: the role of interleukin- 6 in immunity, inflammation and cancer. Trends Mol Med. 2008;14(3):109-119.

77. Sparmann A, Bar-Sagi D. Ras-induced interleukin-8 expression plays a critical role in tumor growth and angiogenesis. Cancer Cell. 2004;6(5):447-458.

78. Tamm I, Kikuchi T, Cardinale I, Krueger JG. Cell-adhesion-disrupting action of interleukin 6 in human ductal breast carcinoma cells. Proc Natl Acad Sci U S A. 1994;91(8):3329-3333.

79. Nagabhushanam V, Solache A, Ting LM, Escaron CJ, Zhang JY, Ernst JD. Innate inhibition of adaptive immunity: mycobacterium tuberculosis-induced IL-6 inhibits macrophage responses to IFNgamma. J Immunol. 2003;171(9):4750-4757.

80. Coppe JP, et al. A human-like senescence-associated secretory phenotype is conserved in mouse cells dependent on physiological oxygen. PLoS One. 2010;5(2):e9188.

81. Coppé JP, et al. Senescence-associated secretory phenotypes reveal cell-nonautonomous functions of oncogenic RAS and the p53 tumor suppressor. PLoS Biol. 2008;6(12):2853-2868.

82. Rodier F, et al. Persistent DNA damage signalling triggers senescence-associated inflammatory cytokine secretion. Nat Cell Biol. 2009;11(8):973-979.

83. Kirkwood TB, Austad SN. Why do we age? Nature. 2000;408(6809):233-238.

84. Parrinello S, Coppe JP, Krtolica A, Campisi J. Stromal-epithelial interactions in aging and cancer: senescent fibroblasts alter epithelial cell differentiation. J Cell Sci. 2005;118(pt 3):485-496

85. Minamino T, et al. A crucial role for adipose tissue p53 in the regulation of insulin resistance. Nat Med. 2009;15(9):1082-1087.

86. Liu D, Hornsby PJ. Senescent human fibroblasts increase the early growth of xenograft tumors via 
matrix metalloproteinase secretion. Cancer Res. 2007;67(7):3117-3126.

87. Campisi J. Cellular senescence: putting the paradoxes in perspective. Curr Opin Genet Dev. 2011;21(1):107-112.

88. Kipling D, Davis T, Ostler EL, Faragher RG. What can progeroid syndromes tell us about human aging? Science. 2004;305(5689):1426-1431.

89. Gasser S, Orsulic S, Brown EJ, Raulet DH. The DNA damage pathway regulates innate immune system ligands of the NKG2D receptor. Nature. 2005;436(7054):1186-1190.

90. Jun JI, Lau LF. Cellular senescence controls fibrosis in wound healing. Aging (Albany NY). 2010;2(9):627-631.

91. Jun JI, Lau LF. The matricellular protein CCN1 induces fibroblast senescence and restricts fibrosis in cutaneous wound healing. Nat Cell Biol. 2010;12(7):676-685.

92. Freund A, Patil CK, Campisi J. p38MAPK is a novel DNA damage response-independent regulator of the senescence-associated secretory phenotype. EMBO J. 2011;30(8):1536-1548.

93. Bhaumik D, et al. MicroRNAs miR-146a/b negatively modulate the senescence-associated inflammatory mediators IL-6 and IL-8. Aging (Albany NY). 2009;1(4):402-411.

94. Orjalo AV, Bhaumik D, Gengler BK, Scott GK, Campisi J. Cell surface-bound IL-1alpha is an upstream regulator of the senescence-associated IL-6/IL-8 cytokine network. Proc Natl Acad Sci US A.
2009;106(40):17031-17036.

95. Coppe JP, Rodier F, Patil CK, Freund A, Desprez PY, Campisi J. Tumor suppressor and aging biomarker p16(INK4a) induces cellular senescence without the associated inflammatory secretory phenotype. J Biol Chem. 2011;286(42):36396-36403.

96. Kipling D, et al. A transcriptomic analysis of the EK1.Br strain of human fibroblastoid keratocytes: the effects of growth, quiescence and senescence. Exp Eye Res. 2009;88(2):277-285.

97. Nelson $G$, et al. A senescent cell bystander effect: senescence-induced senescence. Aging Cell. 2012; 11(2):345-349.

98. Kang TW, et al. Senescence surveillance of pre-malignant hepatocytes limits liver cancer development. Nature. 2011;479(7374):547-551.

99. Yevsa T, Kang TW, Zender L. Immune surveillance of pre-cancerous senescent hepatocytes limits hepatocellular carcinoma development. Oncoimmunology. 2012;1(3):398-399.

100. Hoenicke L, Zender L. Immune surveillance of senescent cells--biological significance in cancer- and non-cancer pathologies. Carcinogenesis. 2012;33(6):1123-1126.

101.Sebastian C, Lloberas J, Celada A. Molecular and cellular aspects of macrophage aging. In: Fulop T, ed. Handbook on Immunosenescence. Doderecht, The Netherlands: Springer Science+Business Media B.V.; 2009:919-945.

102. Guerrero AR, et al. Blockade of interleukin- 6 signaling inhibits the classic pathway and promotes an alternative pathway of macrophage activation after spinal cord injury in mice. J Neuroinflammation. 2012;9:40.

103.Krimpenfort P, Quon KC, Mooi WJ, Loonstra A, Berns A. Loss of p16Ink4a confers susceptibility to metastatic melanoma in mice. Nature. 2001;413(6851):83-86.

104.Wang C, et al. Adult-onset, short-term dietary restriction reduces cell senescence in mice. Aging (Albany NY). 2010;2(9):555-566.

105. Laberge RM, et al. Glucocorticoids suppress selected components of the senescence-associated secretory phenotype. Aging Cell. 2012;11(4):569-578.

106. Kirkland JL, Peterson C. Healthspan, translation, and new outcomes for animal studies of aging. J Gerontol A Biol Sci Med Sci. 2009;64(2):209-212.

107. Voghel G, et al. Cellular senescence in endothelial cells from atherosclerotic patients is accelerated by oxidative stress associated with cardiovascular risk factors. Mech Ageing Dev. 2007;128(11-12):662-671.

108. Price JS, et al. The role of chondrocyte senescence in osteoarthritis. Aging Cell. 2002;1(1):57-65.

109.Bajada S, Marshall MJ, Wright KT, Richardson JB, Johnson WE. Decreased osteogenesis, increased cell senescence and elevated Dickkopf-1 secretion in human fracture non union stromal cells. Bone. 2009;45(4):726-735.

110.Golde TE, Miller VM. Proteinopathy-induced neuronal senescence: a hypothesis for brain failure in Alzheimer's and other neurodegenerative diseases. Alzheimers Res Ther. 2009;1(2):5. 